\title{
LA CONQUISTA DEL DESIERTO, CONFIANZA Y EL PRINCIPIO DE PROXIMIDAD
}

\section{The Conquest of the Desert, Trust, and the Proximity Principle}

\author{
SANTiago Truccone-Borgogno ${ }^{a}$ \\ https://orcid.org/0000-0001-9746-3439 \\ santiago.truccone-borgogno@uni-graz.at \\ ${ }^{a}$ Instituto de Filosofía, Karl-Franzens-Universität Graz, Graz, Austria
}

\begin{abstract}
Resumen
Luego de la Conquista del Desierto, el Estado argentino impuso su ordenamiento institucional a los miembros sobrevivientes de varias comunidades indígenas. De este modo, sus instituciones fueron desplazadas. Esta es una injusticia histórica cuya reparación, en aquel tiempo, requería la restauración de la vigencia de las instituciones indígenas. Sin embargo, no estamos más en 1885 y muchas circunstancias han cambiado. Muchas personas indígenas y no indígenas viven en las mismas ciudades, tienen intereses en las mismas porciones de tierra, e interactúan entre ellos en innumerables formas. Por lo tanto, debe analizarse si, bajo estas condiciones, los reclamos indígenas por recuperar su soberanía siguen siendo válidos. En este trabajo argumentaré que, debido a cambios en las circunstancias, estos reclamos tienen menos fuerza que en el pasado. Por lo tanto, la injusticia histórica no puede ser reparada del mismo modo que en 1885 . No obstante, dada la historia de opresión a la que los indígenas han sido sometidos, el sistema institucional de Argentina tiene que ser reformado de manera tal que su aplicación sobre ellos sea legítima. Propongo tres medidas que contribuyen a conseguir dicho objetivo, a saber, el autogobierno para asuntos internos, la representación en el Senado y el cambio institucional por mayoría simple.
\end{abstract}

Palabras clave: Indígenas; Injusticias históricas; Legitimidad; Tesis de la superación; Waldron.

\begin{abstract}
After the Conquest of the Desert, the State of Argentina forcibly imposed its institutional system over the surviving members of several indigenous communities. In that way, their institutions were ousted. The reparation of this historical injustice, at that time, required the reversion of the indigenous institutions. However, we are not in 1885 anymore, and several circumstances have changed. Many indigenous and non-indigenous persons live in the same cities, have interests in similar portions of
\end{abstract}


land, and interact with each other in an infinite number of ways. Therefore, it should be assessed whether indigenous claims for their sovereignty to be restored are still valid. In this paper, I argue that, owed to changing circumstances, these claims have less normative force than they had in the past. Therefore, those injustices cannot be redressed in the same way as in 1885 . However, I argue that due to the history of oppression indigenous people have suffered, the Argentinian institutional system has to be reformed so that its application over them not being illegitimate. I propose three measures to achieve this aim: self-government over internal affairs, indigenous representatives in the Senate, and institutional change by a simple majority of votes.

Key words: Indigenous People; Historical Injustice; Legitimacy; Supersession thesis; Waldron.

\section{Introducción}

El 5 de octubre de 1878 el Congreso de Argentina sancionó la ley 947 cuyo artículo 1 establecía:

Autorízase al Poder Ejecutivo para invertir hasta la suma de un millón seiscientos mil pesos fuertes en la ejecución de la ley del 13 de Agosto de $1867^{1}$ que disponiendo el establecimiento de la línea de fronteras sobre la margen izquierda de los ríos Negro y Neuquén, previo sometimiento por desalojo de los indios bárbaros de la Pampa, desde el río Quinto y el Diamante, hasta los dos ríos antes mencionados.

Este fue el comienzo de una de las etapas más importantes de lo que se conoce como la "Conquista del Desierto", una serie de campañas militares cuyo objetivo era extender las fronteras de Argentina al sur del territorio. El llamado desierto, sin embargo, no era un desierto en absoluto sino una gran región en la que vivían varias comunidades indígenas. Las campañas militares terminaron en 1885 ante la rendición del lonko Sayhueque (Martínez-Sarasola, 1998, p. 146). ${ }^{2}$ Como resultado de la Conquista del Desierto, más de 60 millones de hectáreas se añadieron al territorio argentino y miles de indígenas fueron asesinados, aprisionados o expulsados fuera de las nuevas fronteras de Argentina (Hasbrouck, 1935, p. 195; Marimán, 2006, p. 113). Muchos indígenas

\footnotetext{
${ }^{1}$ Se refiere a la Ley 215.

${ }^{2}$ El control de las zonas más australes no requirió, en general, de campañas militares. Compañías privadas y pioneros se encargaron de esta tarea (Bandieri, 2000, p. 134).
} 
sobrevivientes, sin embargo, no fueron expulsados sino incorporados de manera forzosa por las autoridades como ciudadanos argentinos (Quijada, 2006, p. 433).

Como sugiere la breve descripción proporcionada, varias injusticias fueron cometidas. Por lo tanto, en aquel tiempo, deberían haber sido reparadas. Sin embargo, más de 130 años han pasado desde que finalizó la Conquista del Desierto y las circunstancias en las que vivimos actualmente son muy diferentes a aquellas de 1885 . Jeremy Waldron ha afirmado que "las injusticias históricas pueden ser superadas (superseded) por cambios en las circunstancias de manera que la situación que fue injusta cuando ocurrió puede coincidir con aquello que la justicia requiere en un tiempo posterior" (2004a, p. 237). ${ }^{3}$ Esta es la tesis de la superación de las injusticias históricas (o, para abreviar, tesis de la superación), que sostiene que incluso si alguna injusticia debería haber sido reparada cuando fue cometida, debido a cambios en las circunstancias, tal reparación puede no ser más requerida.

En este trabajo exploraré ciertos vínculos entre la tesis de la superación y la idea de legitimidad política. Argumentaré que hay buenas razones para afirmar que las instituciones argentinas deben utilizarse para resolver los conflictos que ocurren en su actual territorio, incluso si intereses indígenas están en juego. Sin embargo, para que esto no sea objetable deben ser tomadas medidas específicas que intenten constituir a los miembros de las comunidades indígenas como creadores de las instituciones argentinas.

Para apoyar mi argumento, primero, explicaré el principio de proximidad. Este principio prescribe el deber de formar parte de un Estado con aquellos con los que vivimos en el mismo territorio y con quienes es probable que entremos en conflicto. Segundo, argumentaré que, incluso de acuerdo con este principio, la imposición forzosa del sistema institucional argentino sobre las poblaciones indígenas luego de la Conquista del Desierto fue injusta. Tercero, afirmaré que la superación de esta injusticia podría haber ocurrido solo en la medida en que descansemos en teorías funcionales de la legitimidad. De acuerdo con estas teorías, es legítimo el sistema institucional que proporciona los mejores servicios. Cuarto, explicaré que esta posición funcionalista es muy estrecha dado que no tiene en cuenta la aspiración que tienen los ciudadanos de ser creadores de sus propios sistemas institucionales. Por lo tanto, argumentaré, para que sea superada aquella injusticia histórica es necesario que los miembros de los pueblos indígenas sean

${ }^{3}$ Traducción propia. Todas las traducciones del inglés al castellano son mías. 
puestos en el rol de creadores del sistema institucional que aplica sobre ellos. Quinto, sugeriré que todavía hay obstáculos para que los miembros de los pueblos originarios se involucren en los procesos colectivos de toma de decisiones de Argentina. Por lo tanto, afirmaré que es necesario tomar algunas medidas con el fin de eliminar esas barreras. En este sentido, argumentaré a favor de reconocerles algún nivel de autonomía interna y de que tengan representantes especiales en el Congreso argentino. Asimismo, defenderé que existe un deber moral de no oponerse a la apertura de un proceso que pretenda llevar adelante dichas reformas, si dicho proceso es promovido por una mayoría simple de representantes en el parlamento.

Este asunto, hoy en día, es de mucha importancia práctica. Tómese, por ejemplo, el caso de la ocupación de ciertas parcelas de tierra en el Parque Nacional Lago Mascardi en la Provincia de Rio Negro por parte de la comunidad Mapuche Lof Lafken Winkul Mapu. Los eventos más recientes comienzan con una toma de unas seis hectáreas en 2017 luego de que la Machi de la comunidad designó a esas parcelas como territorio sagrado. Esta incursión es ilegal de acuerdo con la ley argentina ${ }^{4}$ y muchos miembros de la sociedad y oficiales públicos afirman que ocupaciones como esa no deben ser toleradas. Sin embargo, de acuerdo con los miembros de la comunidad Mapuche, dichas parcelas son parte de su Wallmapu ancestral. Para ellos no es una ocupación ilegal dado que el Estado de Argentina ha impuesto sus instituciones sobre ellos de manera injusta y tales leyes continúan siendo ilegítimas. ${ }^{5}$

¿Cómo debemos evaluar tal situación? Este caso es solo un ejemplo. Hay muchos más y en la mayoría de ellos, ante la pregunta ¿quién va a resolver cuestiones que atañen a intereses indígenas? la respuesta -muy probablemente- es: "el Estado argentino". Aún más, ante la pregunta, ¿qué ordenamiento jurídico se aplicará? la respuesta parece ser: "la ley argentina". Por lo tanto, es de vital importancia analizar si está moralmente justificado resolver disputas entre indígenas y no indígenas usando instituciones argentinas.

\footnotetext{
${ }^{4}$ Los artículos 181 y 182 del Código Penal Argentino regulan los delitos de usurpación.

${ }^{5}$ Un resumen de los acontecimientos en dicho lugar realizado por varios medios de comunicación puede verse en [Clarín, 1/09/2020] "Toma de tierras y vecinos asustados que se arman: así es el drama que crece en la Patagonia" https://www.clarin. com/sociedad/toma-tierras-vecinos-asustados-arman-drama-crece-patagonia_0_

PckTKRY0z.html ; [La Nación, 2/09/2020] “¿Qué hay detrás de la toma de tierras en Villa Mascardi?” https://www.lanacion.com.ar/sociedad/que-se-esconde-detras-tomatierras-villa-nid2437923 .
} 


\section{El principio de proximidad}

Entre todos los esquemas de justificación posibles, el principio de proximidad ( $\mathrm{PP})$ parece ser uno de los que aporta razones más fuertes para que los reclamos y asuntos indígenas sean decididos por las instituciones de Argentina. Este principio, kantiano en espíritu, fue desarrollado por Jeremy Waldron en un conjunto de trabajos en los que discute asuntos de legitimidad política y soberanía (1993; 1996; 1999; 2004b; 2006a; 2006b; 2010; 2011; 2018). En líneas generales este principio establece que "Los estados deben formarse entre las personas que ocupan el mismo territorio, tengan o no afinidad entre sí, porque son las que más probabilidades tienen de entrar en conflicto unas con otras" (Waldron, 2011, p. 8).

La justificación de este principio comienza con la idea kantiana de acuerdo con la cual "Es siempre probable que nos encontremos, en principio, junto con otros en los que no confiamos, otros con los que compartimos muy poco en lo que respecta a la cultura, costumbre o religión, otros con los que desacordamos en relación a la justicia" (Waldron 2004b, pp. 58-59). El hecho de que, por un lado, los seres humanos estén agrupados en regiones específicas y que, por el otro, la proximidad genere conflictos, parece requerir un procedimiento para resolver las disputas que puedan surgir. Waldron ilustra este asunto considerando cómo Kant pensó el problema de la adquisición de recursos:

Las personas que viven en las proximidades de otros -de acuerdo con la frase de Kant "inevitablemente lado-a-lado"- probablemente quieran tomar posesión de recursos materiales (tierra o agua, por ejemplo) como parte de su propiedad individual. No obstante, ninguno puede hacerlo sin entrar en conflicto con aquellos que pueden querer la misma parcela de tierra o la misma fuente de agua, y que pueden tener su propia teoría sobre cómo tales cosas deben ser distribuidas de manera adecuada (Waldron, 2010, p. 410).

Hay dos puntos aquí. Primero, los desacuerdos son inevitables entre aquellos que viven cercanos a otros. En el ejemplo, los desacuerdos están relacionados con la adquisición de recursos. Sin embargo, dice Waldron (1996), esto es solo un ejemplo; también se extienden a los principios morales mismos (p. 1553). Cada persona tiene su propia posición en relación a la justicia y los derechos, y es probable que alcancen conclusiones diferentes sobre cómo comportarse. Aun más, incluso si todos comparten un compromiso hacia los mismos valores, pueden alcanzar conclusiones diferentes en relación a cómo especificar 
los principios que surgen de tales valores. Más aún, incluso si existen respuestas correctas sobre, por ejemplo, la apropiación justa de recursos, "no hay base común con la que las partes puedan determinar qué respuestas son correctas" (Waldron, 1996, p. 1550).

El segundo punto es que cuando las personas desacuerdan, el conflicto se generará casi de manera inevitable. Desde un punto de vista kantiano, varias razones explican esto. Recuerda Waldron (1996) que en el estado de naturaleza las personas pueden razonablemente hacer todo lo que crean necesario para promover sus intereses. Además, las personas pueden pelear incluso si creen que la justicia y la moralidad están de su lado (p. 1553). Esto es así, primero, porque hay urgencia en la apropiación de recursos y tal necesidad parece incompatible con esperar hasta que se alcance consenso con otros. Segundo, cuando las personas piensan que la justicia está de su lado es probable que argumenten de manera vehemente y, por lo tanto, la probabilidad de tener conflictos se incrementa (Waldron, 1996, p. 1554).

Sin embargo, la razón crucial por la que el conflicto es problemático está conectada con la idea de libertad y el uso de la fuerza. Los seres humanos tienen interés en ser autores de sus vidas. Esto significa que ellos quieren dirigir sus vidas de acuerdo con sus propias consideraciones. Esta aspiración es expresión del ideal de autonomía personal que, como afirma Raz, "es la imagen de las personas controlando, en algún grado, su propio destino, configurándolo a través de decisiones sucesivas a lo largo de sus vidas" (1986, p. 369). Cumplir con esta aspiración es posible solo si las personas son independientes de los otros. Este derecho a la independencia es, de acuerdo con Kant, nuestro único derecho innato (DR, 63; 6:238). Sin embargo, el problema es que las acciones libremente elegidas pueden impedir que otras personas realicen también acciones libremente elegidas. Por lo tanto, existe la necesidad de identificar bajo qué condiciones o en qué situaciones es permisible afectar el derecho a ser independientes. También hay desacuerdo aquí. El problema, recuerda Waldron, es que "si las personas desacuerdan sobre qué acciones menoscaban incorrectamente la libertad y cuáles no, entonces desacuerdan sobre las ocasiones en que la fuerza puede usarse" (1996, p. 1555). Dado que en el estado de naturaleza no hay mecanismo para resolver conflictos sobre derechos, de acuerdo con Kant, las personas están siempre en una condición de dependencia y falta de libertad. Por fuera del Estado, como afirma Stilz (2019, p. 96) nadie tiene standing exclusivo para determinar e imponer justicia.

Todas las instancias de conflicto y desacuerdo parecen requerir un mecanismo para lidiar con ellas. Incluso si dos contrincantes en una 
disputa tratan de proceder de buena fe es posible que el segundo no comparta la decisión del primero. Aún más, incluso si se llega a algún acuerdo, no hay garantía de que cada participante mantenga el trato. Como afirma Stilz (2009, p. 51), cada contrincante todavía es dependiente de la voluntad privada del otro, representando esto, en sí mismo, una situación de no libertad. Lo que se necesita es un acuerdo en función del cual las decisiones que se tomen sean impuestas y que represente una reflexión detallada sobre la materia. Como afirma Waldron "esto es lo que puede ofrecer el Estado: un cuerpo de leyes bien pensado y formado que se hará cumplir de manera unívoca, de una manera que no represente simplemente un compromiso o vector entre fuerzas individuales" (2006b, p. 193).

El Estado ofrece la posibilidad de lidiar con desacuerdos y proporciona no solo una base para solucionarlos respetando el sentido de justicia de las personas, sino también "insiste en que [el problema del desacuerdo] debe resolverse entre aquellos entre quienes surge" (Waldron, 2010, p. 412). De aquí que, como afirma Kant, no cualquier Estado debe formarse sino solo aquel que refleje una voluntad omnilateral, es decir la voluntad unificada de todos (DR, p. 125 [6:314]). Se requiere, en otras palabras, entrar en la sociedad civil y proceder con aquellos con los que estamos lado-a-lado a partir de condiciones establecidas por el derecho positivo. Es un deber moral que le debemos a aquellos con los que es probable que entremos en conflicto (Waldron, 1999, pp. 58-59). Por lo tanto, es algo que cada contrincante en un conflicto puede hacer cumplir. Entrar en el Estado es, para resumir, algo que le debemos a aquellos con los que vivimos lado-a-lado dado que no hay otro modo de establecer condiciones a partir de las cuales cada persona pueda ser independiente.

\section{El principio de proximidad y la Conquista del Desierto}

Luego de la Conquista del Desierto, 8000 familias indígenas continuaban viviendo en los territorios del sur de Argentina y sus miembros fueron incorporados de manera forzosa a la autoridad argentina como ciudadanos argentinos (Quijada, 1999, p. 695; 2006, p. 433). La pregunta es si esta incorporación estuvo justificada.

De acuerdo con el PP, aquellos que viven lado-a-lado deben entrar en una comunidad política donde los desacuerdos y conflictos sean resueltos utilizando el derecho positivo y otras instituciones públicas. Desde que, luego de la Conquista del Desierto, las personas indígenas y no indígenas tenían que vivir lado-a-lado, y muchos conflictos y desacuer- 
dos existían entre ellos, la oposición de los miembros de los pueblos indígenas a ser parte de las instituciones argentinas parece injustificada. Es decir, los miembros de las comunidades indígenas perecen haber tenido el deber de entrar en la sociedad civil con los ciudadanos no indígenas de Argentina. Parece que, de acuerdo con el PP, la imposición del sistema jurídico argentino sobre los indígenas sobrevivientes a la Conquista del Desierto no fue injusta.

A decir verdad, no creo que el PP haya requerido que, en 1885, las personas indígenas queden sujetas a las instituciones argentinas. Primero, la situación de los miembros de muchas comunidades indígenas no era como la de los anarquistas obstinados que no quieren ser parte del Estado. Anna Stilz afirma que "para calificar como "Estado", de acuerdo con [su] posición, una institución debe poseer (i) autogobierno colectivo vinculante y (ii) la capacidad de hacer efectivas las decisiones sobre los casos en disputa" (2019, p. 14). De acuerdo con esta concepción no es preciso decir que los miembros de las comunidades indígenas adoptaron la postura irresponsable de no querer ser partes de una comunidad institucionalizada. Todo lo contrario, los miembros de las comunidades indígenas tenían sus propias instituciones. Por ejemplo, en la totalidad del Wallmapu, el pueblo Mapuche tenía mecanismos institucionalizados de solución de conflictos (Kollgtun, Füuta, Trawun, Wepin), espacios institucionales para poder desarrollarlos (Juntas, Parlamentos, Xaxüm), e incluso agentes específicos con la autoridad de hacer cumplir las decisiones (Lonko, Werken, Machi) (Marimán, 2006, pp. 65-101). De acuerdo con esta posición, las comunidades indígenas no negaron el deber general de ser parte del Estado. Por el contrario, ellos reconocían tal obligación, pero rechazaban el deber particular de entrar en un Estado específico: Argentina. ${ }^{6}$

No obstante, podría objetarse que el PP requería formar un Estado común dado que tanto Argentina como las comunidades indígenas

${ }^{6}$ Un evaluador anónimo ha objetado que si los sistemas institucionales de las poblaciones indígenas no hubiesen sido de calidad suficiente para que el derecho a la independencia de cada miembro sea garantizado es posible que el Estado de Argentina no hubiese tenido el deber de respetar su soberanía. Creo que el evaluador tiene razón al afirmar que una calidad deficiente de las instituciones indígenas podría haber justificado que el Estado de Argentina no deba respetar la soberanía de los pueblos originarios. Sin embargo, de aquí no se sigue que hubiese estado permitido incorporar a esos indígenas a las instituciones del Estado interventor. Una tesis más plausible, creo, es pensar que tal intervención podría haberse permitido o, incluso, haber sido requerida pero solo para ayudar a que dichas instituciones indígenas sean capaces de garantizar el derecho a la independencia de los miembros de su propia comunidad. Véase Applbaum (2019, cap. 4). 
tenían reclamos territoriales sobre las mismas porciones de tierra. Por lo tanto, continúa la objeción, ante esta disputa jurisdiccional ninguna de las partes estaba obligada a aceptar la autoridad de la otra sino que ellas debían formar un Estado común. ${ }^{7}$

La respuesta a esta objeción comienza notando que, hasta aproximadamente 1885 , las comunidades indígenas que estoy considerando y el Estado argentino ocupaban territorios diferentes. De hecho, es conocida la famosa Zanja de Alsina, una fosa gigante cuya construcción comenzó en 1876 bajo la administración de Nicolás Avellaneda con el objetivo de contener a la población indígena a lo largo de lo que se consideraba la frontera sur del país (Rock, 2019, p. 175). Dada esta separación territorial no es claro que a finales del siglo XIX las comunidades indígenas y no indígenas hayan tenido el deber de formar un Estado común. Aun si había conflictos, no diferían de aquellos que podían existir entre, por ejemplo, los estados de Argentina y de Chile. Es posible pensar que ambas comunidades podrían haber continuado viviendo permanentemente separadas, por ejemplo, una a cada lado de la Zanja de Alsina o, incluso, del Rio Negro. De hecho existía una larga tradición de realizar tratados entre, por un lado, (primero) la Corona española o (luego) distintas provincias argentinas y, por otro lado, los pueblos indígenas, reconociendo autonomía a estos últimos. ${ }^{8}$

El hecho de que ambas comunidades hayan tenido su asiento principal de vida en territorio separado parece aportar razones a favor de que, dentro de cada territorio, dichos grupos hayan tenido al menos lo que se conoce como derechos de ocupación. El derecho de ocupación sobre un territorio determinado incluye dos incidentes. Primero, la libertad de residir y hacer uso de las prácticas imperantes en ese territorio. Segundo, un derecho (claim-right) a no ser expulsado del territorio ni ser obstaculizado en el disfrute de las prácticas allí imperantes (Stilz, 2019, p. 35).

Los derechos de ocupación no garantizan el derecho a excluir a otros a no ser que los extranjeros que incursionan en el territorio ocupado dañen los planes de vida, la cultura y las prácticas económicas de los locales (Stilz, 2019, p. 73). En ausencia de tal afectación es posible que la construcción de un Estado común que incluya a indígenas y no indígenas sea no solo no objetable sino también, a la luz del PP, requerida. Con relación a la Conquista del Desierto, el problema es que la incursión realizada por el Estado argentino en los territorios hasta ese momento ocupados por los indígenas alteró de manera drástica los planes de

\footnotetext{
${ }^{7}$ Agradezco a los evaluadores anónimos por presionar sobre este punto.

${ }^{8}$ Véase Levaggi (1995).
} 
vida de las poblaciones indígenas. Por ejemplo, mientras Julio Roca era presidente de Argentina, se sancionó la ley 1628 de 1885, llamada Ley de Premios Militares. De acuerdo con esta ley, las tierras previamente ocupadas por los pueblos indígenas tenían que ser transferidas a los expedicionarios del desierto (Bandieri, 2000, p. 155). Esta política generó un desplazamiento de los miembros de los pueblos indígenas a las zonas que, hasta ese momento, eran consideradas poco productivas y de escaso valor para ser usadas para la agricultura y ganadería. Dada la injusticia que rodea a estos hechos y, sobre todo, porque los planes de vida de los miembros de los pueblos originarios fueron radicalmente afectados por la Conquista del Desierto hay razones para pensar que los pueblos indígenas tenían un derecho justificado a excluir al Estado de Argentina de su territorio.

No obstante, se podría afirmar que, aun si tal derecho de exclusión estaba justificado, una vez concluida la Conquista del Desierto, dadas las nuevas circunstancias, estaba justificada la imposición forzosa del ordenamiento institucional argentino sobre los indígenas sobrevivientes de dichas campañas militares. Para esto puede ser de ayuda pensar en una situación hipotética en la que el Estado argentino era perfectamente (o, al menos, casi) justo e impuso sus instituciones sobre los miembros renuentes de los pueblos indígenas. ${ }^{9}$

Kant repudiaba la imposición forzosa del propio orden institucional a aquellos miembros de otros pueblos con los que no había perspectiva de unión común (DR, p. 87 [6:266]). Más allá de Kant, uno podría preguntarse de todos modos si está justificado -y, en tal caso, bajo qué condiciones- incorporar de manera forzosa a los miembros de otro pueblo al propio sistema institucional. Una de estas condiciones parece requerir que aquellos sobre quienes se aplicará el sistema institucional, como mínimo, tengan la posibilidad de participar en el diseño de dicho sistema. ${ }^{10}$ Si esto no sucede es difícil ver cómo tal sistema institucional puede considerarse también como producto de la voluntad de estos sujetos, como requiere el PP. Por lo tanto, en el caso que nos ocupa, deberíamos preguntarnos si los miembros indígenas sobrevivientes de la Conquista del Desierto tuvieron alguna posibilidad de participar en el proceso de diseño institucional argentino.

\footnotetext{
${ }^{9}$ El escenario es similar al de Simmons (2016, p. 56) en el que los Estados Unidos anexiona una parte de Méjico e impone sus instituciones sobre los ciudadanos mejicanos.

${ }^{10}$ Charles Beitz (1989, p. 109) llama interés en reconocimiento al interés en no ser excluido de los roles públicos ni de la participación en los procesos de toma de decisión colectiva.
} 
Sobre este punto es de utilidad comenzar con el Artículo 67, Inciso 15 de la Constitución de Argentina de 1853 que establecía entre las atribuciones del Congreso: "Proveer a la seguridad de las fronteras; conservar el trato pacífico con los indios y promover la conversión de ellos al catolicismo". ${ }^{11}$ Tal inciso fue el resultado de discusiones entre conservadores y liberales, ambos grupos no indígenas que, por un lado, no consideraron los puntos de vista indígenas sobre el asunto y, por el otro, subordinaron sus intereses a los de los no indígenas. Como recuerda Nino (1992, p. 123), incluso los liberales argumentaron solo desde el punto de vista no indígena dado que, para ellos, la conversión de los indígenas al catolicismo era el único modo posible de alcanzar un trato pacífico con ellos. Existen varios puntos a considerar sobre esta disposición. Sin embargo, por ahora, basta con resaltar que los indígenas no tuvieron la posibilidad de participar en este proceso.

El asunto es aún peor cuando se considera específicamente el modo en que fueron incorporados a las instituciones de Argentina. Luego de la Conquista del Desierto, dos posiciones fueron defendidas en el debate parlamentario de 1885. Por un lado, un parlamentario sostuvo la posición, fundada en el modelo de los Estados Unidos, de que los indígenas tenían que ser alojados en reservas (Quijada, 1999, p. 692). Sin embargo, el punto de vista que predominó fue el opuesto. Los indígenas de Argentina debían ser considerados ciudadanos argentinos porque nacieron en territorio argentino y eso es lo que establecían la Constitución y las leyes. Desde el punto de vista jurídico, ellos fueron incorporados de manera forzosa como ciudadanos argentinos (Quijada, 2006, p. 433). ${ }^{12}$ Sin embargo, los miembros de los pueblos indígenas no tuvieron la posibilidad de participar ni siquiera en este asunto. Por lo tanto, la imposición forzosa del sistema jurídico argentino sobre ellos fue injusta, incluso a las luces del PP. En aquel tiempo la soberanía indígena sobre su territorio debería haber sido restaurada.

\section{Soberanía y la tesis de la superación}

En la sección anterior argumenté que la imposición forzosa del sistema institucional argentino sobre los indígenas sobrevivientes de

\footnotetext{
${ }^{11}$ Las reformas constitucionales de 1860, 1866 y 1898 mantuvieron esta cláusula. La de 1949 la derogó. Sin embargo, fue restaurada en la de 1957 para ser, nuevamente, derogada en la de 1994.

${ }^{12}$ Esto no quiere decir que no haya habido reservas sino solo que esta idea no fue adoptada como política general. Sobre la historia de algunas reservas indígenas, véase Aguerre (2008, pp. 54-65).
} 
la Conquista del Desierto fue injusta. Sin embargo, no estamos más en 1885 y muchas circunstancias han cambiado. Muchas personas indígenas y no indígenas viven en las mismas ciudades, barrios, tienen intereses en las mismas porciones de tierra, y sus modos de vida están entrelazados en innumerables formas. Por lo tanto, se debe analizar si, bajo estas condiciones, los reclamos indígenas por recuperar su soberanía siguen siendo válidos.

Los reclamos por soberanía pueden ser entendidos como reclamos por legislar sobre un territorio particular. En este sentido, se pretende que las instituciones indígenas tengan la autoridad para resolver todos los conflictos que puedan tener lugar en sus tierras ancestrales. Así, la soberanía se asocia con reclamos por autodeterminación. ${ }^{13} \mathrm{Si}$ consideramos, como ejemplo, el caso Mapuche, esto significa que en todos los asuntos que ocurran en el Puelmapu (su territorio ancestral dentro de las fronteras argentinas), las instituciones indígenas deberían tener la autoridad de decidir. Estoy pensando en las disputas sobre derechos de propiedad, de ocupación, o -directamente- en desacuerdos entre personas indígenas y no indígenas en todas sus interacciones. Con relación a estas disputas hay una razón bastante evidente para afirmar que tienen que ser resueltas de acuerdo con las instituciones indígenas. La razón es que tales instituciones fueron desplazadas de manera injusta a finales del siglo XIX y, en aquel tiempo, tal soberanía debería haberse restaurado.

El problema, sin embargo, es que las circunstancias cambian con el tiempo y los cambios en las circunstancias pueden superar o anular las injusticias históricas. La tesis de la superación descansa sobre la afirmación de que los derechos son circunstancialmente sensibles en el sentido de que pueden estar justificados en circunstancias $\mathrm{C}_{1}$ pero no bajo un conjunto de circunstancias diferentes $\mathrm{C}_{2}$ (Waldron, 2004b, p. 68). Antes de 1885, muchos grupos indígenas eran comunidades soberanas reguladas por sus propias instituciones. Bajo circunstancias $\mathrm{C}_{1}$ presentes en aquel tiempo $\mathrm{T}_{1}$, una respuesta adecuada a la imposición forzosa del sistema institucional argentino era restaurar la vigencia de las instituciones indígenas. Esta respuesta es correcta en un tiempo $\mathrm{T}_{1}$ en las circunstancias $\mathrm{C}_{1}$. Sin embargo, en $\mathrm{T}_{2}$, esto es, entre 1885 y 2021, las circunstancias cambiaron a $\mathrm{C}_{2}$. Por tanto, la pregunta es si en el 2021, esto es, en un tiempo $\mathrm{T}_{3}$, con circunstancias $\mathrm{C}_{2}$ las instituciones

${ }^{13}$ Los reclamos por soberanía también pueden interpretarse como demandas por autonomía, es decir, por legislar exclusivamente sobre ciertas personas y asuntos internos. En la sección 7 realizaré algunas consideraciones al respecto. 
indígenas tienen que ser restauradas. Este sería el caso si la injusticia no fue superada. Así, los desacuerdos y conflictos que tengan lugar en su territorio deberían ser resueltos por instituciones indígenas. En cambio, si la injusticia fue superada, tal derecho a ser soberanos sobre su territorio ancestral no debe ser restaurado y las decisiones tomadas por las instituciones argentinas en relación a las comunidades indígenas podrían ser legítimas.

\section{La superación de los reclamos indígenas por soberanía}

Permítaseme asumir que las comunidades indígenas que sufrieron la imposición del sistema institucional argentino en el pasado sobrevivieron al presente. ¿Es este hecho suficiente para afirmar que las disputas y conflictos relacionados con asuntos indígenas deben ser resueltos por las instituciones indígenas latentes? O, en cambio, ¿deben ser resueltos por las instituciones del Estado de Argentina?

Hay dos discusiones aquí. Primero, nos topamos con una situación en la que dos sistemas institucionales pretenden decidir sobre el mismo tipo de conflicto en el mismo territorio. Por lo tanto, la pregunta es si existe alguna razón para preferir alguno. La segunda discusión surge si se responde de manera afirmativa a la primera pregunta. ¿Cómo podemos decidir cuál de los dos sistemas institucionales debe tener autoridad para decidir?

En relación al primer asunto hay al menos dos razones para elegir entre dos sistemas institucionales que intentan tener aplicación en el mismo territorio sobre la misma cuestión. La primera razón se conecta con los problemas de coordinación. Incluso si ambos sistemas actúan en función de los mismos principios de justicia, dado que ellos pueden elegir diferentes políticas para alcanzar los mismos resultados, hay razones de eficiencia para optar por uno (Waldron, 1993, pp. 23-24). La segunda razón está directamente conectada, dice Waldron, con las razones por las que-de acuerdo con el PP kantiano- las personas tienen el deber de entrar en la sociedad civil. Formar un Estado y proceder bajo las condiciones del derecho positivo es un medio para resolver conflictos y desacuerdos. Waldron dice que "el interés moral en reducir tales contiendas proporciona una razón para que todos apoyemos a la misma organización, y eso nos da a cada uno una razón para unirnos y apoyar a cualquier organización a la que los otros apoyen y se hayan unido" (1993, p. 23). Si no nos uniésemos al mismo Estado y diferentes instituciones tuviesen la autoridad de decidir sobre la misma disputa, los desacuerdos y conflictos que proporcionaban razones para formar 
el Estado resurgirían. Por esta razón, si dos o más sistemas tuvieran competencia sobre el mismo territorio y en relación al mismo asunto, las personas no estarían viviendo en condiciones de libertad.

Ahora bien, ¿ cómo podemos decidir entre dos (o más) sistemas institucionales que compiten por resolver el mismo asunto? El siguiente párrafo de Waldron resume su posición:

Si un par de sistemas institucionales rivales, $\mathrm{L}_{1}$ y $\mathrm{L}_{2}$, en un territorio $\mathrm{T}$ son tales que la mayoría de la población de $\mathrm{T}$ acordaría en ser gobernada por $\mathrm{L}_{1}$ en lugar de por $\mathrm{L}_{2}$ si les preguntásemos, y si todos en $\mathrm{T}$ conocen esto sobre los dos sistemas, entonces parece que $\mathrm{L}_{1}$ es claramente la opción predominante como el sistema al que se le debe lealtad por consideraciones de justicia, si tal lealtad es debida a algún sistema institucional (Waldron, 1993, p. 26).

El argumento parece descansar en la idea de consenso, algo extraño para alguien como Waldron quien argumenta en contra de tales tesis transaccionales sobre legitimidad política. Sin embargo, en su argumento, el consenso es solo un modo de evaluar la efectividad de un sistema institucional particular. Para él, "la mera existencia de una institución como dominante e indiscutida puede ser suficiente para establecer su predominio, sea popularmente apoyada o no" (Waldron, 1993, p. 25). El consentimiento, para él, es solo uno de los modos de acuerdo con los cuales el predominio de un sistema institucional es identificado. Para Waldron, el sistema institucional predominante es aquel que sirve de mejor modo a los intereses de aquellos sobre los que se aplica. Él ve a la soberanía, el Estado y al derecho como una brigada de bomberos. "Si los servicios están disponibles para todos no importa de quién es la brigada, o a qué tradiciones corresponde, o quién la puso en funcionamiento o cómo; lo que importa es que la tenemos y funciona" (Waldron, 2006a, p. 24).

Si pensamos nuevamente en los reclamos indígenas en Argentina, para determinar qué sistema debe resolver los conflictos y desacuerdos debemos analizar cuál de los dos sistemas que compiten suministran (comparativamente) los mejores servicios, en el sentido de ser capaces de proporcionar justicia de manera más efectiva. Esta es una pregunta complicada dado que los sistemas institucionales indígenas no están vigentes. Ellos han sido desplazados por el argentino. Por lo tanto, es difícil hacer la comparación requerida. De todos modos, un hecho parece hablar a favor del uso del sistema argentino. La complejidad de las sociedades contemporáneas y el número de conflictos e interacciones 
entre indígenas y no indígenas requiere ahora, a diferencia de en 1885, un sistema institucional común y sofisticado para que puedan ser resueltos. El Estado de Argentina se ha mostrado relativamente capaz de hacer esto. De hecho, aunque más no sea por una cuestión estratégica, muchos integrantes de los pueblos indígenas recurren a las instituciones del Estado argentino no solo para aprovisionarse de bienes públicos sino también para canalizar muchos reclamos por tierras. ${ }^{14}$ Como en otros Estados, las instituciones de Argentina se han desarrollado con los conflictos y desacuerdos que tienen que arreglar, y sus instituciones son relativamente capaces de lidiar con los retos del mundo moderno. El Estado argentino cuenta con un sistema institucional que, en general, protege derechos humanos básicos, respeta mínimamente los requisitos de justicia distributiva y proporciona de manera relativamente adecuada diferentes bienes públicos.

Estas consideraciones no aplican a los sistemas institucionales indígenas desplazados. Desde que el sistema institucional indígena está "suspendido" no ha tenido la oportunidad de desarrollarse. Así, aunque más no sea por una cuestión de efectividad actual, el sistema institucional de Argentina funciona mejor que los sistemas institucionales indígenas suspendidos. Por lo tanto, el primero debe ser preferido. Si el sistema institucional argentino debe ser preferido, de acuerdo con esta posición, la injusticia referida a la imposición forzosa del ordenamiento institucional argentino sobre los miembros de los pueblos indígenas fue superada.

\section{Libertad, alienación y superación parcial}

La sección anterior concluye que es legítimo el sistema institucional que proporciona los mejores servicios. No hay algo así como "la ley de alguien". Debemos considerar, afirma Waldron, "[a la ley] a la luz de un aparato funcional que está allí y disponible en un territorio, no como posesión o patrimonio de alguna persona o grupo particular" (2006a, p. 24). Esta posición funcionalista es compatible con la imposición forzosa de sistemas institucionales sobre las personas. Waldron reconoce este punto. Sin embargo, no piensa que el problema sea serio. Él afirma que la importancia de la justicia y la necesidad de tener una institución política que la persiga son suficientes para afirmar que una organización particular puede imponerse a sí misma sobre cierto territorio (Waldron, 1993, p. 27). La historia, de acuerdo con él, no

${ }^{14}$ Véase Ramos \& Delrio (2005). 
tiene un gran valor. Todo lo que importa es que el sistema proporcione las condiciones para que las personas sean capaces de ser autoras de sus propias vidas. En la medida en que exista un sistema y funcione, su aplicación generalizada a todos está justificada.

Waldron tiene razón al afirmar que, dado que la proximidad genera conflictos, las personas no tienen la opción de ser o no parte del Estado. Por lo tanto, que alguien no haya consentido el sistema jurídico que se le aplica es -por sí mismo- irrelevante para asuntos de legitimidad. ${ }^{15}$ Sin embargo, del hecho de que el consentimiento no sea necesario para la legitimidad de un sistema institucional no se sigue que lo único que importe sea cómo funciona dicho sistema. En la disputa entre teorías del deber natural versus teorías transaccionales de legitimidad, Waldron (2018, p. 549) critica a las transaccionales por pasar por alto la plausibilidad de las intuiciones que subyacen al enfoque estructural-funcionalista. ${ }^{16}$ La posición de Waldron -creo- ignora la plausibilidad de las intuiciones que subyacen a las posiciones históricotransaccionales de legitimidad. La posición funcional no puede capturar las dos dimensiones relevantes implicadas en la noción de legitimidad política. Para que un sistema institucional sea legítimo no es suficiente con proporcionar los mejores servicios a sus ciudadanos; también es necesario que las instituciones sean producto de aquellos sobre los que se aplican. Si esto no sucede, como argumentaré debajo, es difícil ver cómo la aspiración de los ciudadanos en ser autores de sus propias vidas puede ser satisfecha.

Un modo de dar cuenta de ambas clases de consideraciones es distinguiendo, como hace Anna Stilz, entre dos clases de intereses de la ciudadanía. "Como 'tomadores' institucionales, los individuos tienen intereses en la protección de sus derechos, en la justicia distributiva y en los bienes públicos que proporciona el Estado" (Stilz, 2016, p. 100). Esta es la dimensión del sistema institucional sobre la que Waldron pone el acento cuando afirma que debemos preferir el sistema que sirva mejor a los intereses de aquellos sobre los que se aplica. Esta dimensión de la ciudadanía, en la que somos tomadores del sistema institucional, es incorporada correctamente por las teorías funcionales de la legitimidad. Sin embargo, la perspectiva de Stilz $(2015$, p. 8) también incorpora una segunda dimensión. Esta se refiere al interés que tienen los ciudadanos en crear y ser coautores del sistema institucional en el que viven. Este

\footnotetext{
${ }^{15}$ De aquí no se sigue que el sistema institucional pueda ser impuesto de cualquier modo. Gracias a un evaluador anónimo por sugerirme esta cualificación.

${ }^{16}$ Se refiere a Simmons (2016).
} 
interés en ser "creadores" requiere que las instituciones puedan ser atribuidas a aquellos sobre los que se aplican. Cuando este no es el caso, las personas se encuentran alienadas del sistema institucional. ${ }^{17}$

Hay varias razones por las que es valioso que el sistema institucional sea creación de sus ciudadanos. Una de las más importantes se conecta con la idea de autonomía personal. Las personas, como afirmé más arriba, tienen la aspiración de gobernar sus vidas de acuerdo con sus propias decisiones. Sin embargo, la amenaza a que cada persona pueda ser autora de su vida también puede provenir del Estado. El problema surge cuando las instituciones no pertenecen a los ciudadanos. En tal caso, cada decisión que es impuesta sobre ellos usando el derecho es ajena al individuo. En tal situación, por lo tanto, el sistema institucional no respeta la aspiración fundamental de cada individuo de ser autor de su vida.

Cuando los ciudadanos son creadores del sistema institucional, por un lado, como afirma Stilz, el proyecto colectivo les importa "de un modo en que el éxito de la empresa conjunta se conecta con su propio bienestar" (2016, p. 113). Por el otro lado, se cambia la relación entre los ciudadanos y el sistema institucional. Como afirma Stilz, "aquí, el Estado no es más un poder avasallante y ajeno, sino una herramienta que les permite realizar de manera más efectiva sus propios compromisos" (2019, p. 107). Debido a estas razones, el interés de cada persona en ser creadora del sistema institucional es importante. Solo cuando tal interés es satisfecho no hay alienación entre los ciudadanos y el sistema institucional que se aplica sobre ellos.

En función de las consideraciones anteriores, para que un sistema institucional sea legítimo tiene que satisfacer los intereses que los sujetos tienen en ser sus tomadores y creadores. El interés en ser tomadores se satisface cuando el Estado es mínimamente justo. En cambio, el interés en ser creadores se satisface cuando las instituciones no son ajenas a aquellos sobre quienes se aplican. Por lo tanto, para afirmar que la injusticia consistente en la imposición forzosa del sistema institucional argentino sobre las poblaciones indígenas fue superada debe mostrarse que son satisfechos no solo sus intereses como tomadores sino también como creadores del sistema institucional que se les aplica.

Con relación a este asunto, es dudoso que, en la historia de Argentina, los intereses de los indígenas como creadores del sistema institucional hayan sido (mínimamente) satisfechos. Luego de que los miembros de los pueblos indígenas fueran incorporados como

${ }^{17}$ Véase Lu (2017, p. 190). 
ciudadanos argentinos, pero antes de 1920, sus puntos de vista fueron escasamente considerados en los debates públicos. Entre 1920 y 1930 comenzó a desarrollarse un esquema de reconocimiento de las injusticias históricas perpetradas en contra de las poblaciones indígenas. Sin embargo, el modo de reparar tales injusticias fue a través de medidas paternalistas que no consideraban sus puntos de vista. Fue solo luego de 1966 y, con mayor fuerza, con el retorno de la democracia en 1983 que los grupos indígenas comenzaron a formar redes con el objetivo de trascender y de disputar la autoridad en las decisiones conectadas a sus intereses (Lenton, 2010, p. 72). No obstante, incluso en aquellos asuntos que conciernen especialmente a sus intereses territoriales ancestrales, los pueblos indígenas no son generalmente consultados en los procesos de decisión colectiva (Svampa, 2016, p. 357).

De acuerdo con esta descripción, la situación de muchas comunidades indígenas en Argentina puede ser caracterizada como un caso de lo que Stilz (2015, p. 20) llama fallas de legitimidad subjetiva garantizadas, en las que los intereses de un grupo particular de personas como creadoras de un sistema institucional son persistentemente insatisfechos como resultado de una historia de relaciones políticas opresivas. En estas situaciones, sus reclamos para que su sistema institucional suspendido sea restaurado parecen tener más fuerza. El problema es que, aunque el sistema institucional argentino pueda satisfacer mínimamente los intereses como tomadores de los indígenas y no indígenas, no satisface de manera adecuada los intereses que los miembros de varias comunidades indígenas tienen en ser sus creadores.

Si las consideraciones anteriores son correctas es posible que la imposición forzosa del sistema jurídico argentino sobre los miembros de los pueblos indígenas no haya sido superada, incluso si sus intereses como tomadores están satisfechos. Esto no implica que la soberanía indígena deba ser restaurada. Puede ser un caso de lo que Lukas Meyer y Timothy Waligore llaman superación parcial. La superación parcial ocurre cuando, aunque no existe un deber de volver a la situación inmediatamente anterior a la injusticia original, "los reclamos históricos todavía tienen algún peso" (Meyer \& Waligore, 2016, p. 11). Por lo tanto, puede ser verdadero que el Estado de Argentina no tenga el deber de restaurar la soberanía "suspendida" de las comunidades indígenas. Esto sería así, como he argumentado en la sección 5, dado que la complejidad de la sociedad actual y el número de conflictos e interacciones entre personas indígenas y no indígenas requiere un esquema institucional común para su solución. Por lo tanto, si la disputa es entre dos sistemas en pugna, a saber, el argentino vigente y el indígena, y el argentino -a 
diferencia de los sistemas indígenas suspendidos- se ha mostrado capaz de proteger derechos básicos, satisfacer ciertas demandas de justicia distributiva y de proveer bienes públicos, entonces el sistema argentino vigente debe preferirse.

Por supuesto, parte de la razón por la que los sistemas indígenas no se han desarrollado en el tiempo del modo en que lo hizo el argentino se debe a la misma injusticia de haber sido desplazado a la fuerza. De todos modos, este punto no es óbice para afirmar que la injusticia pasada fue superada. Como afirma Waldron, "el cambio en las circunstancias al que refiere la tesis de la superación pueden incluir cambios que son el producto causal inmediato de la misma injusticia que se objeta" (2004a, p. 243). Esto es así dado que la justicia depende de las circunstancias y, como he argumentado más arriba, las circunstancias presentes son tales que hacen que el sistema argentino proporcione los mejores servicios tanto a no indígenas como a indígenas.

Sin embargo, la superación de la injusticia histórica es solo parcial. El Estado de Argentina todavía tiene que dar cuenta del interés que los miembros de las comunidades indígenas tienen en ser creadores del sistema institucional. Para esto, no es necesario que los miembros de los pueblos indígenas "se sientan", por decirlo de algún modo, creadores de tal sistema. Todo lo que es necesario es que dicho sistema los trate como tales. Como afirma Seleme (2010, p. 91) no se es autor (o creador) del sistema institucional por participar activamente en él sino que es el mismo diseño institucional el que debe configurar a sus ciudadanos como tales. En este sentido, para que el interés en tanto creadores del sistema institucional sea satisfecho, las instituciones deben configurar a los miembros de los pueblos indígenas como tales. Por lo tanto, se debe analizar de qué modo es posible configurar a los miembros de las comunidades indígenas con una larga historia de opresión como creadores del sistema institucional argentino.

\section{Aspectos de la ciudadanía y medidas de reconciliación}

A manera de ejemplo de cómo un sistema institucional puede dar cuenta de ambos intereses de la ciudadanía (como tomadora y como creadora) en relación a grupos históricamente oprimidos veamos un aspecto de la Reforma Constitucional de 1994. ${ }^{18}$ Antes de 1994,

${ }^{18}$ Esto es solo un ejemplo sobre cómo, en una oportunidad, ambos intereses en relación a los miembros de los pueblos indígenas- pueden haberse satisfecho (parcialmente). Por sí mismo, el caso bajo análisis no puede mostrar completamente de qué modo y en qué medida los intereses conectados con la ciudadanía son 
la vigencia del Artículo 67, Inciso 15 (reseñado arriba) contribuía no solo a la subordinación de los intereses de los miembros de los pueblos indígenas a los no indígenas sino también a la alienación institucional de los primeros. Dicho inciso, vale la pena recordar, establecía entre las atribuciones del Congreso: "Proveer a la seguridad de las fronteras; conservar el trato pacífico con los indios y promover la conversión de ellos al catolicismo".

Sin embargo, este inciso de la Constitución Nacional cambió en 1994 al actual Artículo 75 inciso 17, según el cual se encuentran entre las atribuciones del Congreso:

Reconocer la preexistencia étnica y cultural de los pueblos indígenas argentinos. Garantizar el respeto a su identidad y el derecho a una educación bilingüe e intercultural; reconocer la personería jurídica de sus comunidades, y la posesión y propiedad comunitarias de las tierras que tradicionalmente ocupan; y regular la entrega de otras aptas y suficientes para el desarrollo humano; ninguna de ellas será enajenable, transmisible ni susceptible de gravámenes o embargos. Asegurar su participación en la gestión referida a sus recursos naturales y a los demás intereses que los afecten. Las provincias pueden ejercer concurrentemente estas atribuciones.

El actual Artículo 75 Inciso 17 es más beneficioso para las comunidades indígenas que el antiguo Artículo 67 Inciso 15. Esto es, por supuesto, importante. Es relevante que la nueva provisión constitucional reconozca derechos mínimos a las comunidades indígenas y, de ese modo, satisfaga de mejor modo los intereses que sus miembros tienen como tomadores. Sin embargo, este no es el punto en el que me quiero detener. Un cambio interesante es que, a diferencia de la provisión constitucional anterior, la nueva fue el resultado de (entre otras cosas) considerar los puntos de vistas indígenas. Aún más, varios representantes de los pueblos originarios pudieron expresar sus posiciones durante la convención constitucional de $1994 .{ }^{19}$ Sin embargo, la existencia de un proceso de toma de decisiones colectivas con dichas características es todavía insuficiente. Para que el interés de los miembros de las comunidades indígenas en ser creadores del sistema institucional sea satisfecho

satisfechos. Sin embargo, el análisis es útil para identificar qué dirección deben tomar las reformas institucionales si intentan dar cuenta de tales intereses.

${ }^{19}$ Diario de Sesiones de la Convención Constituyente de 1994 (Comisión de Nuevos Derechos y Garantías, Reunión 28/3/10, 11 de agosto de 1994, pp. 4065-4067). 
no alcanza con que no existan barreras formales para su acceso a roles públicos y a la participación en los procedimientos de toma de decisión colectiva. Si ese fuera el caso, la Reforma Constitucional de 1994 implicaría que ambos intereses de la ciudadanía han sido satisfechos.

El problema surge del siguiente modo. En la medida en que el interés de los miembros de los pueblos originarios en ser creadores de las instituciones no sea satisfecho, las instituciones argentinas les son ajenas. Para que tal interés sea satisfecho no tiene que haber barreras para que los miembros de las comunidades indígenas tengan la posibilidad de involucrarse en los procesos de toma de decisiones colectivas. El asunto es que, dada la historia de opresión, los indígenas no tienen razones para confiar en los demás. Como afirma Timothy Waligore, "cuando un grupo le ha negado a otro un disfrute seguro de sus derechos en el pasado, puede ser razonable (o no irrazonable) que los sucesores de las víctimas quieran mantenerse al margen de un contacto extensivo con los sucesores de los perpetradores" (2009, p. 34). Esta falta de confianza es, de hecho, una barrera que imposibilita o, al menos, dificulta que los miembros de los pueblos indígenas participen en los procesos decisorios institucionales argentinos. ${ }^{20}$ Por lo tanto, el Estado de Argentina debe eliminar dichas barreras si pretende colocar a los indígenas en el rol de creadores del sistema institucional.

Dado que las barreras para que los miembros de las comunidades indígenas sean creadores del sistema institucional provienen del hecho de que ellos no tienen razones para confiar en otros, las medidas específicas a ser tomadas deben promover dicha confianza. ${ }^{21}$

La primera medida para promover una confianza mínima entre indígenas y no indígenas en Argentina consiste en reconocerle a los primeros ciertas formas de autonomía o autogobierno entendido como su derecho a gobernar sobre asuntos internos. Quiero proceder con cautela aquí. Como nota Waldron (2010) el argumento fundado en la identidad en favor del autogobierno asume dos afirmaciones. La primera (a) es que ciertas especificidades de una determinada cultura son de suma importancia para el bienestar individual. La segunda (b) es que cuando una cultura determinada tiene relevancia para el bienestar personal

${ }^{20}$ Véase Kropff (2005).

${ }^{21}$ Las medidas que propondré requieren -probablemente- modificar la parte orgánica de la Constitución. Como afirma Gargarella (2013, pp. 179-185), gran parte del problema de las reformas constitucionales recientes en relación al "asunto indígena" se debe a una desatención en las tensiones que existen entre, por un lado, expandir ciertos derechos (en la parte dogmática de la Constitución); y, por el otro, dejar casi intacta la parte referida a la organización del poder. 
de sus miembros se requiere que ellos tengan un grado significativo de control político sobre los asuntos del grupo (p. 401). ${ }^{22}$ La afirmación (a) es innegable. Como resalta Meyer, "las personas pueden valorar su membresía en una comunidad como un fin debido a las oportunidades únicas que la comunidad les ofrece" (2001, p. 271). Los grupos no solo son instrumentalmente valiosos para el bienestar de sus miembros, sino que, también, pueden ser intrínsecamente valiosos para ellos. Por ejemplo, los miembros de un grupo pueden darle valor intrínseco al hecho de vivir en una sociedad justa o tolerante, al mismo tiempo que pueden considerar que vivir en una sociedad justa o tolerante contribuye al fomento de otros valores como la estabilidad (Meyer, 2001, p. 270).

Sin embargo, el asunto es si tal interés en mantener el bienestar del grupo lo máximo posible puede fundar reclamos por autogobierno (b). Mi respuesta es que depende del caso. Si un sistema institucional puede mantener el bienestar de diferentes grupos, sus propiedades intrínsecamente valiosas, y sus miembros pueden participar de manera efectiva en los procesos de toma de decisión colectiva, entonces, no veo razón por la que dichos grupos deberían tener la oportunidad de formar una unidad política (separada). Sin embargo, el caso de los grupos que sufren una larga historia de opresión es diferente. El sistema institucional argentino actual no solo no fue capaz de proteger esas opciones que son valiosas para los miembros de grupos indígenas, sino que también cuenta con barreras para que ellos se involucren activamente en las instituciones del Estado de Argentina. En situaciones como esta, hay -por tanto- razones para que los grupos históricamente oprimidos tengan cierto grado de autogobierno. Esto sería un modo no solo de que ellos puedan mantener esas propiedades que consideran valiosas sino también una manera de restaurar la confianza en la población mayoritaria y en las instituciones de Argentina. Al restaurar tal confianza, ellos pueden empezar a considerar al Estado como un proyecto conjunto valioso. De este modo, las barreras para que las comunidades indígenas participen en los procesos de toma de decisiones colectiva pueden ser mitigadas. Por lo tanto, el interés en ser creadores del sistema institucional estará más cerca de ser satisfecho. ${ }^{23}$

${ }^{22}$ Margalit y Raz (1990, pp. 454-461) proporcionan una justificación instrumental para los reclamos de autogobierno fundados en la identidad nacional. Bajo ciertas condiciones, argumentan, el derecho a autogobierno fundamenta ciertos reclamos por autodeterminación sobre un territorio específico.

${ }^{23}$ Haber sufrido una larga historia de opresión es uno de los factores que da legitimidad a los reclamos de los grupos históricamente oprimidos y que los distingue de los de otros grupos que también pueden sentirse poco representadas en los procesos 
En aras de la autonomía interna, por ejemplo, los pueblos indígenas pueden también tener alguna forma de parlamento institucionalizado con competencia para diagramar ciertas políticas legalmente reconocidas por el Estado argentino. ${ }^{24}$ Esta es solo una de muchas posibilidades para articular alguna forma de autogobierno. Puede haber otras. Las comunidades indígenas Mapuche, por ejemplo, cuentan con un sistema para resolver conflictos basados en el Azmapu. El Azma$p u$ es un conjunto de normas y reglas que regulan las relaciones entre las personas y el ambiente. Como sistema de normas, ellas cuentan con distintas soluciones (reparaciones, castigos, etc.) ante su violación (Cárdenas-Llamacán, 2019, p. 102). De esta manera sería posible pensar también en algún tipo de reconocimiento legal a alguna forma de justicia comunitaria. Sin embargo, como muestra la experiencia latinoamericana, sería de mucha importancia articular modos adecuados de coordinación entre los sistemas de justicia "ordinarios" y "comunitarios" (Ramírez, 2017, pp. 112-114). Entre los retos de coordinación entre sistemas podemos encontrar la posible tensión entre ciertas interpretaciones indígenas de los derechos colectivos con la idea-más individualista-de respeto por los derechos humanos. ${ }^{25}$

La segunda medida consiste en reformar la Cámara de Senadores del Congreso de la Nación de modo que las comunidades indígenas (o una federación de comunidades indígenas) puedan tener representantes allí. A primera vista, esta medida parece muy radical. Sin embargo, esto no es así. Argentina es un Estado federal, como lo establece el Artículo 1 de la Constitución. Ser un Estado federal significa que "hay una división de poderes, constitucionalmente definida y protegida, entre un gobierno central cuyas decisiones aplican a todos los miembros

de toma de decisiones colectivas. No hay barreras basadas en la desconfianza, por ejemplo, para que un libertario o un descendiente de italianos se involucre en los asuntos parlamentarios.

${ }^{24}$ Para una descripción de como, por ejemplo, el pueblo Mapuche se organiza políticamente en Argentina actualmente, ver Cañuqueo, Kropff y Pérez (2015). Dado que la comunidad Mapuche tiene miembros tanto en Argentina como en Chile, dichos parlamentos podrían devenir en una especie de parlamento transestatal en el que los miembros de las comunidades indígenas puedan canalizar sus demandas por autonomía en todo lo que consideran su territorio (el Wallmapu). Al considerar el caso de los Saami en Escandinavia, Lukas Meyer proporciona un análisis detallado sobre cómo estas medidas pueden ser justificadas (2001, pp. 291-295).

${ }^{25} \mathrm{El}$ establecimiento de protecciones externas que intentan promover la equidad entre grupos puede ayudar. En cambio, debe ser rechazada la existencia de restricciones internas que limiten el derecho de los miembros del grupo a cuestionar y revisar sus prácticas y autoridades (Kymlicka, 1995, p. 37). 
del Estado, y varios gobiernos subestatales cuyas decisiones se aplican solamente a un segmento de la población de dicho Estado" (Weinstock, 2001, p. 75).

En el caso de Argentina, el poder está dividido entre el gobierno central, veintitrés provincias y la Ciudad Autónoma de Buenos Aires. ${ }^{26}$ Dada esta circunstancia, el poder legislativo está divido en dos cámaras. Por un lado, la Cámara de Diputados y, por el otro, la de Senadores. Los miembros de la Cámara de Diputados representan a la totalidad de la población de Argentina. En cambio, en la Cámara de Senadores, cada provincia cuenta con tres representantes que defienden sus intereses como provincia.$^{27}$ Por lo tanto, el actual sistema institucional argentino es sensible a los intereses que un grupo especifico de personas tienen como grupo. La propuesta, entonces, es expandir el ámbito de aquellos que pueden, en el Congreso de Argentina, defender sus intereses como grupo. Esto requiere reformar la Cámara de Senadores para que las comunidades indígenas cuenten con, al menos, tres senadores que, como los representantes de las provincias, puedan defender y argumentar a favor de sus intereses específicos.

Realizar tal reforma incrementaría no solo la confianza de las poblaciones indígenas en las instituciones de Argentina sino también sería un modo de reconocer -y también reparar- la injusticia histórica consistente en haber diseñado un sistema institucional sin considerar la existencia de un grupo específico de personas que, como las provincias argentinas, tenían intereses específicos en el momento en que el poder fue dividido institucionalmente. ${ }^{28}$ Después de todo, el inciso 17 del Artículo 75 de la Constitución establece que es deber del Congreso "Reconocer la preexistencia étnica y cultural de los pueblos indígenas argentinos". Por lo tanto, no debería resultar extraño que los grupos indígenas puedan tener asientos en el Senado para canalizar sus demandas del mismo modo en que lo tienen las provincias. ${ }^{29}$

${ }^{26}$ Conforme a los artículos 121 y 129 de la Constitución.

${ }^{27}$ Esto está regulado especialmente en los artículos 44, 45 y 54 de la Constitución.

${ }^{28} \mathrm{El}$ federalismo argentino fue formado bajo un proceso que se suele caracterizar como "unión" (coming together) (Føllesdal, 2018, para. 2), dado que varias provincias preexistían y se unieron para organizar al Estado federal, como fue establecido en las Constituciones de 1853-1860.

${ }^{29}$ En otros países tales como Nueva Zelanda, como recuerda Applbaum, "aquellos que se identifican como maoríes y eligen colocar sus nombres en una circunscripción electoral diferente completan los asientos en función de los votos maoríes" (Applbaum, 2019 , p. 177). Uno podría pensar en una medida similar para ser implementada en la Cámara de Diputados. Los indígenas podrían tener la posibilidad de registrarse en una circunscripción electoral especial para poder votar por representantes indígenas. 
La tercera medida es análoga a una presentada por Thomas Pogge, quien nos hace pensar en un país en el que sus fundadores se dan una Constitución que contiene una cláusula que prescribe que, luego de la muerte de cualquier propietario, sus posesiones son heredadas por su hijo varón de mayor edad. La Constitución también prescribe que cualquier cambio de dicha cláusula requiere una mayoría de $2 / 3$ votos obtenidos de un referéndum. Sin embargo, luego de varios años de discusión, un grupo de mujeres aboga por la sustitución de dicha cláusula por una alternativa en función de la cual, luego de la muerte de cualquier propietario, sus posesiones son heredadas por su descendiente de mayor edad (Pogge, 2004, p. 124). El argumento que usa este grupo de mujeres a favor del cambio no se refiere a la injusticia del precepto constitucional, que de hecho existe, sino a que "en el momento fundacional y por más de un siglo desde entonces, dicen, las mujeres fueron completamente excluidas del proceso político" (Pogge, 2004, p. 125). En el ejemplo de Pogge, hay un referéndum y la propuesta de las mujeres obtiene el $60 \%$ de los votos. De acuerdo con la vieja provisión constitucional, esta mayoría es insuficiente para cambiar la cláusula sobre la herencia dado que se requieren $2 / 3$ de votos. Luego de este resultado, continúa Pogge, el grupo de mujeres que aboga por tal reforma concluye que el $40 \%$ de la población que votó en contra de la propuesta tiene una razón moral para abstenerse de usar su poder para bloquear la reforma constitucional dado que "una mayoría simple merece ganar debido a que la provisión constitucional que intentan cambiar, que establece una asimetría en función del sexo [en las reglas de herencia], fue atrincherada de una manera injusta" (Pogge, 2004, p. 125). Esto es, en una situación en las que las mujeres tenían prohibido participar en el diseño institucional.

Creo que un argumento análogo puede ser aplicado a la situación de los indígenas en Argentina. Cuando el poder fue institucionalmente dividido en las Constituciones de 1853 y 1860, los miembros de los pueblos indígenas no fueron parte de las convenciones constitucionales y, como argumenté más arriba, sus intereses no fueron adecuadamente considerados. Esta situación, sumada a que ellos pertenecen a grupos que, como las provincias, son preexistentes al Estado argentino, justifica que puedan tener representantes específicos en el parlamento. Sin embargo, dicha modificación en el sistema institucional seguramente requerirá una reforma constitucional. El Artículo 30 de la Constitución requiere $2 / 3$ de votos de los miembros del Congreso para abrir tal proceso de reforma. Sin embargo, en el momento en que tal cláusula fue sancionada los miembros de los pueblos indígenas no podían participar. 
El problema es que, si una mayoría simple quisiera hacer el sistema institucional más inclusivo con relación a los pueblos indígenas, una minoría tendría un poder de veto obtenido de manera injusta toda vez que fue adquirido en circunstancias donde existían importantes barreras para la participación de las poblaciones indígenas. Por lo tanto, parece que hay razones para no oponerse a una apertura del proceso constitucional si una mayoría simple quiere comenzar con dicho proceso con el fin de que los miembros de los pueblos originarios puedan participar en el diseño institucional. Este es un deber moral negativo que consiste en no usar un poder de veto adquirido gracias a un atrincheramiento injusto de derechos. Como afirma Pogge, "si una mayoría favorece un cambio constitucional, una minoría no debería usar el poder de veto que pueda tener gracias a un atrincheramiento injusto que bloquee la voluntad mayoritaria" (Pogge, 2004, p. 127). Cumplir con este deber expresa que el sistema institucional argentino, al menos de ahora en más, debe ser el producto de todos, incluso de las poblaciones indígenas.

\section{Conclusión}

En este trabajo he cuestionado la legitimidad de las instituciones argentinas en relación a los miembros de los pueblos indígenas que viven en su actual territorio. Argumenté que para que un sistema institucional sea legítimo debe dar cuenta de los intereses que la ciudadanía tiene tanto como tomadora y como creadora. Comencé analizando el principio de proximidad que afirma que los Estados deben ser formados entre aquellos que ocupan el mismo territorio y es probable que entren en conflicto entre sí. Argumenté que, incluso a la luz de tal principio, la imposición forzosa del ordenamiento institucional argentino sobre las poblaciones indígenas luego de la Conquista del Desierto fue injusta. Sin embargo, he argumentado que, actualmente, tanto los miembros de las comunidades indígenas como no indígenas deben ser parte del mismo sistema institucional. Las instituciones de Argentina pueden ocupar tal rol dado que se encuentran en una posición ventajosa para satisfacer los intereses que tanto indígenas como no indígenas tienen como tomadores de tal sistema.

Sin embargo, el Estado argentino es deficiente en relación a los miembros de las comunidades indígenas. Sus intereses como creadores no son (mínimamente) satisfechos dado que existen ciertas barreras que impiden su involucramiento en los procesos de toma de decisiones colectivas. Para arreglar tal carencia he propuesto algunas medidas que 
intentan promover la confianza entre los indígenas y la población no indígena (y el Estado). Realizar alguna de estas medidas permitiría que los miembros de las comunidades indígenas sean colocados en el rol de creadores del sistema institucional argentino. En la medida en que este no sea el caso, la injusticia histórica consistente en la imposición forzosa del sistema institucional sobre ellos no habrá sido superada. Por lo tanto, la aplicación de las instituciones argentinas sobre los miembros de las comunidades indígenas sigue siendo ilegítima. ${ }^{30}$

\section{Bibliografía}

Aguerre, A. (2008). Genealogías de familias tehuelches-araucanas de la Patagonia central y meridional argentina. Editorial de la Facultad de Filosofía y Letras de la Universidad de Buenos Aires.

Applbaum, A. (2019). Legitimacy. Harvard University Press.

Bandieri, S. (2000). Ampliando las fronteras: la ocupación de la Patagonia. En M. Lobato (Dir.), Nueva historia argentina: el progreso, la modernización y sus límites (1880-1916) (vol. 5., pp. 119-177). Sudamericana.

Beitz, C. (1989). Political equality. Princeton University Press.

Cañuqueo, L., Kropff, L., y Pérez, P. (2015). A la sombra del estado: comunalización indígena en parajes de la precordillera de Río Negro, Argentina. Revista del Museo de Antropología, 8(2), 159-170.

Cárdenas-Llancaman, M. (2019). Azmapu: una propuesta normativa mapuche. Administración Pública y Sociedad (APyS), 8, 97-109.

Føllesdal, A. (2018). Federalism. En E. Zalta (ed.), The Stanford Encyclopedia of Philosophy (Summer Ed.). https://plato.stanford. edu/archives/sum2018/entries/federalism/

Gargarella, R. (2013). Latin American constitutionalism, 1810-2010: The

${ }^{30}$ Presenté versiones anteriores de este trabajo en el workshop "Extending Justice in Space and Time" del Centro de Investigaciones Filosóficas (2019), en el Seminario "Populisme, Conceptes, Subjetes y Governs" de la Universidad de Girona (2020), y en distintos seminarios de la Universidad de Graz. Agradezco a los participantes de estos eventos por sus críticas y comentarios. Estoy particularmente agradecido con Lukas Meyer, Timothy Waligore, Rodrigo Sánchez-Brígido, Wolfgang Benedek, Romina Rekers, Julio Montero, Francisco Garcia-Gibson, Marcelo de Araujo, Laura García-Portela, Eduardo Rivera-López, Alessandro Pinzani, Lucas Misseri y Anna Bugajska. Agradezco también a dos referís anónimos y a los editores de esta revista por sus valiosos comentarios. El artículo es mejor gracias a todos ellos. Este trabajo es parte de una investigación realizada en el contexto del proyecto "Supersession of Historical Injustice and Changed Circumstances" financiado por el Fondo Austríaco para la Ciencia (FWF) bajo el subsidio de investigación: P 30084. 
engine room of the constitution. Oxford University Press. Hasbrouck, A. (1935). The conquest of the desert. The Hispanic American Historical Review, 15(2), 195-228.

Kant, I. (1991). DR: The doctrine of right. En The metaphysics of morals (introduction, translation, and notes by Mary Gregor). Cambridge University Press.

Kropff, L. (2005). Activismo mapuche en Argentina. En P. Dávalos (Ed.), Pueblos indigenas, estado y democracia (pp. 103-132). CLACSO.

Kymlicka, W. (1995). Multicultural citizenship. Clarendon Press.

Lenton, D. (2010). Política indigenista argentina: una construcción inconclusa. Anuário Antropológico, 35(1), pp. 57-97. https://doi. org/10.4000/aa.781

Levaggi, A. (1995). Tratados celebrados entre gobiernos argentinos e indios del sur de Buenos Aires, Santa Fe, Córdoba y Cuyo (18101852). Revista de Historia del Derecho "Ricardo Levene", 30, 87165.

Lu, C. (2017). Justice and Reconciliation in World Politics. Cambridge University Press.

Margalit, A. \& Raz, J. (1990). National self-determination. The Journal of Philosophy, 87(9), 439-461.

Marimán, P. (2006). Los mapuche antes de la conquista militar chilenoargentina. En P. Marimán, S. Caniuqueo, J. Millalén, \& R. Levil, i... Escucha, Winka...! Cuatro ensayos de historia nacional mapuche y un epílogo sobre el futuro (pp. 53-127). Lom.

Martínez-Sarasola, C. (1998). Los hijos de la tierra: Historia de los indigenas argentinos. Emecé.

Meyer, L. (2001). Transnational autonomy: Responding to historical injustice in the case of the Roma peoples. International Journal on Minority and Group Rights, 8(2/3), 263-301.

Meyer, L., \& Waligore, T. (2016). Superseding historical injustice and changed circumstances. FWF Project Proposal.

Nino, C. (1992). Fundamentos de derecho constitucional. Astrea.

Pogge, T. (2004). Historical wrongs: The two other domains. En L. Meyer (Ed.), Justice in time (pp. 117-134). Nomos.

Quijada, M. (1999). La ciudadanización del "indio bárbaro": Políticas oficiales y oficiosas hacia la población indígena de la Pampa y la Patagonia, 1870-1920. Revista de Indias, 59(217), 675-704.

Quijada, M. (2006). De mitos nacionales, definiciones cívicas y clasificaciones grupales: Los indígenas en la construcción nacional argentina de los siglos XIX a XXI. En W. Ansaldi (Ed.), Calidoscopio latinoamericano (pp. 426-450). Ariel. 
Ramírez, S. (2017). Diversidad en los modos de gestionar la conflictividad: Profundizando el derecho al acceso a la justicia. Revista sobre acceso à justica e direitos nas Américas ABYA YALA, 2, 107-121.

Ramos, A. \& Delrio, W. (2005). Trayectoria de oposición. Los mapuches y tehuelches frente a la hegemonía de Chubut. En C. Briones (Comp.), Cartografías argentinas (pp. 73-107). Antropofagia.

Raz, J. (1986). The morality of freedom. Oxford University Press.

Rock, D. (2019). The British in Argentina: Commerce, Settlers \& Power 1800-2000. Macmillan.

Seleme, H. (2010). La legitimidad como autoría. Revista Brasileira de Filosofía, 234, 73-99.

Simmons, J. (2016). Boundaries of authority. Oxford University Press.

Stilz, A. (2009). Liberal loyalty. Princeton University Press.

Stilz, A. (2015). Decolonization and self-determination. Social Philosophy \& Policy Foundation, 32(1), 1-24. https://doi.org/10.1017/ S0265052515000059

Stilz, A. (2016). The value of self-determination. En D. Sobel, P. Vallentyne, \& S. Wall (Eds.). Oxford studies in political philosophy (vol. 2, pp. 98-127). Oxford University Press.

Stilz, A. (2019). Territorial sovereignty. Oxford University Press.

Svampa, M. (2016). Debates latinoamericanos. Edhasa.

Waldron, J. (1993). Special ties and natural duties. Philosophy and Public Affairs, 22(1), 3-30.

Waldron, J. (1996). Kant's legal positivism. Harvard Law Review, 109(7), $1535-1566$.

Waldron, J. (1999). The dignity of legislation. Cambridge University Press.

Waldron, J. (2004a). Settlement, return, and the supersession thesis. Theoretical Inquiries in Law, 5(2), 237-268.

Waldron, J. (2004b). Redressing historic injustice. En L. Meyer (Ed.), Justice in time (pp. 55-77). Nomos.

Waldron, J. (2006a). Supersession and sovereignty. New York University Public Law and Legal Theory Working Papers. Paper 406. http:// lsr.nellco.org/nyu_plltwp/406

Waldron, J. (2006b). Kant's theory of the state. En P. Kleingeld (Ed.), Toward perpetual peace and other writings on politics, peace, and history / Immanuel Kant (pp. 179-200). Yale University Press.

Waldron, J. (2010). Two conceptions of self-determination. En S. Besson, \& J. Tasioulas (Eds.), The philosophy of international law (pp. 397-413). Oxford University Press.

Waldron, J. (2011). The principle of proximity. Public Law \& Legal 
Theory Research Paper Series, Working Paper 11-08. http://ssrn. com/abstract $=1742413$

Waldron, J. (2018). Boundaries of authority. Philosophical Review, $127(4), 545-550$.

Waligore, T. (2009). Cosmopolitan right, indigenous peoples, and the risk of cultural interaction. Public Reason 1(1), 27-56.

Weinstock, D. (2001). Towards a normative theory of federalism. International Journal of Social Sciences, 167, 75-83.

Recibido el 28 de octubre de 2020; revisado el 12 de marzo de 2021; aceptado el 25 de marzo de 2021. 